Research Article

\title{
Algebraic Relations among Four Types of Right Semi-Tensor Product
}

\author{
Nating Chen $\mathbb{D}^{1,2}$ Menglei Lin $\mathbb{D}^{1,2}$ and Yiliang $\mathrm{Li} \mathbb{D}^{3}$ \\ ${ }^{1}$ School of Mathematics and Statistics, Minnan Normal University, Zhangzhou, Fujian 363400, China \\ ${ }^{2}$ Institute of Meteorological Big Data-Digital, Minnan Normal University, Zhangzhou, Fujian 363400, China \\ ${ }^{3}$ School of Mathematics, Shandong University, Jinan, Shandong 250100, China
}

Correspondence should be addressed to Menglei Lin; menglei36@126.com

Received 6 May 2021; Accepted 7 June 2021; Published 14 June 2021

Academic Editor: Renming Yang

Copyright ( $\odot 2021$ Nating Chen et al. This is an open access article distributed under the Creative Commons Attribution License, which permits unrestricted use, distribution, and reproduction in any medium, provided the original work is properly cited.

In this paper, algebraic relations among four kinds of right semi-tensor product (STP) are discussed. Firstly, this paper provides definitions of right STPs, consisting of the first right matrix-matrix STP, the second right matrix-matrix STP, the first right matrixvector STP, and the second right matrix-vector STP. Secondly, relations among these right STPs are proposed. Finally, the main results show the convertibility of these right STPs.

\section{Introduction}

Cheng product, also called semi-tensor product (STP), was first introduced by Prof. Cheng [1]. A significant breakthrough of STP is that it overcomes the dimension barrier, which limits the development of conventional matrix product. The first type of STP is called left matrix-matrix (MM) STP, which expends the dimension of each matrix by right multiplying the identity matrix. Here, the matrix product refers to the Kronecker product. Thanks to the left MM STP, a Boolean network can be converted into a linear discrete-time form, which stimulates the development of Boolean networks [2-4]. In addition, the left MM STP also plays an important role in finite game [5-7], fuzzy systems $[8,9]$, and graph theory $[10,11]$.

However, the left MM STP loses its ability in discussing dimension-varying linear systems because the result of left MM STP of a matrix and a vector is a matrix instead of a vector. Thus, the second type of STP, called left matrixvector (MV) STP, was proposed in [12]. Compared with the left MM STP, the left MV STP expends the dimension of vector by right multiplying a column vector whose elements are 1. Based on the left MV STP, dimension-varying linear systems were modeled [13]. Besides, the solution and stability of continuous-time dimension-varying linear systems were studied in [14]. References $[15,16]$ considered state dimensions of dimension-varying discrete linear systems.

Due to the importance of left MM STP and left MV STP, the authors in [17] explored more general STPs, which help to expand the possible applications of STP. In the following, the left MM STP and left MV STP are denoted by left MM-1 STP and left MV-1 STP. By right multiplying $J_{n}=(1 / n) 1_{n \times n}$, the left MM-2 STP and left MV-2 STP are defined in [18]. Here, $1_{n \times n}$ is an $n \times n$ dimensional matrix, whose elements are 1. Relations among these four kinds of left STP are discussed in [19].

By changing the way of dimension expansion, the authors in [17] defined four corresponding right STPs, including right MM-1 STP, right MM-2 STP, right MV-1 STP, and right MV-2 STP. However, relations between these right STPs are not studied. Hence, this paper reviews definitions of right STP and discusses relations among these right STPs. The main results show the convertibility of these right STPs.

The rest of this paper is organised as follows. Section 2 introduced definitions of four kinds of right STP. Relations among these right STPs are investigated in Section 3. Section 4 gives some concluding remarks.

Before ending this section, we provided a list of notations, most of which can be found in $[20,21]$. 
(i) $\mathscr{M}_{m \times n}$ contains all $m \times n$ dimensional matrices. The $i$-th column (row) of matrix $M$ is denoted by $\operatorname{Col}_{i}(M) \quad\left(\operatorname{Row}_{i}(M)\right)$. Particularly, $M_{i j}=$ Row $_{i}$ $\left(\mathrm{Col}_{j}(M)\right)$.

(ii) $\mathscr{V}_{p}$ includes all $p$ dimensional column vectors.

(iii) $I_{n}$ is the $n$ dimensional identity matrix.

(iv) $1_{n \times n}=\left(1_{n}, 1_{n}, \ldots, 1_{n}\right)$, where $1_{n}=\sum_{i=1}^{n} \operatorname{Col}_{i}\left(I_{n}\right)$.

(v) $0_{m \times n}$ is an $m \times n$ dimensional zero matrix.

(vi) $A^{T}$ is the transpose of matrix $A$.

$($ vii) $\otimes$ is the Kronecker product

\section{Definitions of Right STP}

In this section, definitions of right STP are introduced. To begin with, the right STP of two matrices is proposed. In this paper, STP refers to right STP.

Definition 1 (see $[12,17])$. Let $A \in \mathscr{M}_{m \times n}$ and $B \in \mathscr{M}_{p \times q}$. Suppose $t=[n, p]$ is the least common multiple of $n$ and $p$.

(1) The first right MM STP (MM-1 STP) of $A$ and $B$, denoted by $A \rtimes_{1} B$, is defined as

$$
A \rtimes_{1} B=\left(I_{t / n} \otimes A\right)\left(I_{t / p} \otimes B\right) .
$$

(2) The second right MM STP (MM-2 STP) of $A$ and $B$, denoted by $A \rtimes_{2} B$, is defined as

$$
A \rtimes_{2} B=\left(J_{t / n} \otimes A\right)\left(J_{t / p} \otimes B\right)
$$

where $J_{k}=(1 / k) 1_{k \times k}$ and $k=(t / n)$ or $k=(t / p)$.

Remark 1. If $n=p$, then $A \rtimes_{1} B=A \rtimes_{2} B=A B$. It means that MM-1 STP and MM-2 STP are generalizations of the conventional matrix product. In addition, MM-1 STP and MM-2 STP not only keep main properties of conventional matrix product available but add some new properties such as certain commutativity [12].

Given a matrix $A \in \mathscr{M}_{m \times n}$ and a vector $x \in \mathscr{V}_{n}$. Then, the conventional matrix product of $A$ and $x$ can be viewed as a linear mapping on $\mathscr{V}_{n}$. However, MM-1 STP and MM-2 STP cannot be regarded as linear mappings because results of MM-1 STP and MM-2 STP are matrices instead of vectors. Thus, other definitions of right STP, as extensions of linear mappings, are provided.

Definition 2 (see $[12,17])$. Let $A \in \mathscr{M}_{m \times n}$ and $x \in \mathscr{V}_{r}$. Suppose $s=[n, r]$ is the least common multiple of $n$ and $r$.

(1) The first right MV STP (MV-1 STP) of $A$ and $x$, denoted by $A \vec{\rtimes}_{1} x$, is defined as

$$
A \vec{\rtimes}_{1} x=\left(I_{s / n} \otimes A\right)\left(1_{s / r} \otimes x\right) .
$$

(2) The second right MV STP (MV-2 STP) of $A$ and $x$, denoted by $A \vec{\rtimes}_{2} x$, is defined as

$$
A \vec{\rtimes}_{2} x=\left(J_{s / n} \otimes A\right)\left(1_{s / r} \otimes x\right)
$$

Definition 3. Given $A \in \mathscr{M}_{m \times n}$ and $B \in \mathscr{M}_{p \times q}$. Suppose $t=$ $[n, p]$ is the least common multiple of $n$ and $p$. Then,

$$
\begin{aligned}
& A \vec{\rtimes}_{1} B=\left(I_{t / n} \otimes A\right)\left(1_{t / p} \otimes B\right), \\
& A \vec{\rtimes}_{2} B=\left(J_{t / n} \otimes A\right)\left(1_{t / p} \otimes B\right) .
\end{aligned}
$$

\section{Algebraic Relation among Four Types of STP}

In this section, algebraic relations among four types of STP are discussed. The following three cases are considered: (1) STP of two vectors, (2) STP of matrices and vectors, and (3) STP of two matrices.

3.1. TP of Two Vectors. Firstly, algebraic relations among STPs of two column vectors are concerned.

Theorem 1. Let $x \in \mathscr{V}_{n}$ and $y \in \mathscr{V}_{p}$ be two column vectors. Then, the following results hold:

$$
\begin{gathered}
\text { (1) } x \vec{\rtimes}_{1} y=x \rtimes_{1} y=y \otimes x \\
\text { (2) } x \vec{\rtimes}_{2} y=x \rtimes_{2} y=(1 / p) \\
y=\left(y_{1}, y_{2}, \ldots, y_{p}\right)
\end{gathered}
$$$$
\text { (2) } x \vec{\rtimes}_{2} y=x \rtimes_{2} y=(1 / p) \sum_{i=1}^{p} y_{i}\left(1_{p} \otimes x\right), \quad \text { where }
$$

Proof. (1) The correctness of the first item is obvious, so the proof is omitted. (2) In the light of the definition of MM-2 STP, one calculates

$$
x \rtimes_{2} y=\left(J_{p} \otimes x\right) y=\frac{1}{p}\left(1_{p \times p} \otimes x\right) y=\frac{1}{p}\left(1_{p}^{T} \otimes 1_{p} \otimes x\right) y=\frac{1}{p} \sum_{i=1}^{p} y_{i}\left(1_{p} \otimes x\right) .
$$

In addition, it is easy to see that $x \vec{\rtimes}_{2} y=x \rtimes_{2} y$ holds for any column vectors $x$ and $y$.

The following results are about algebraic relation among STPs of two row vectors.
Theorem 2. Let $x^{T} \in \mathscr{V}_{n}$ and $y^{T} \in \mathscr{V}_{p}$ be two column vectors. Then, the following results hold:

$$
\begin{aligned}
& \text { (1) } x \rtimes_{1} y=x \otimes y \\
& \text { (2) } x \vec{\rtimes}_{2} y=x \vec{\rtimes}_{1} y=\sum_{i=1}^{n} x_{i} y \text {, where } x=\left(x_{1}, x_{2}\right. \text {, } \\
& \left.\ldots, x_{n}\right)
\end{aligned}
$$


(3) $x \rtimes_{2} y=(1 / n) 1_{n}^{T} \otimes\left(x \vec{\rtimes}_{2} y\right)=(1 / n) 1_{n}^{T} \otimes\left(x \vec{\rtimes}_{1} y\right)=$ $(1 / n) \sum_{i=1}^{n} x_{i}\left(1_{n}^{T} \otimes y\right)$, where $x=\left(x_{1}, x_{2}, \ldots, x_{n}\right)$

Proof

(1) The correctness of the first item is obvious, so the proof is omitted.
(2) By computing directly, one can see that $x \vec{x}_{1} y=$ $x\left(1_{n} \otimes y\right)=\sum_{i=1}^{n} x_{i} y$ holds

(3) From the definition of MM-2 STP, we get

$$
x \rtimes_{2} y=x\left(J_{n} \otimes y\right)=\frac{1}{n} x\left(1_{n \times n} \otimes y\right)=\frac{1}{n} x\left(1_{n}^{T} \otimes 1_{n} \otimes y\right)=\frac{1}{n} 1_{n}^{T} \otimes\left(x\left(1_{n} \otimes y\right)\right)=\frac{1}{n} 1_{n}^{T} \otimes\left(x \vec{\rtimes}_{2} y\right) .
$$

Combined with the first two items, one obtains $x \rtimes_{2} y=$ $(1 / n) 1^{T} \otimes\left(x \vec{\rtimes}_{1} y\right)=(1 / n) 1_{n}^{T} \otimes\left(\sum_{i=1}^{n} x_{i} y\right)=(1 / n) \sum_{i=1}^{n} x_{i}$ $\left(1_{n}^{T} \otimes y\right)$.

3.2. STP of Matrices and Vectors. In this subsection, algebraic relations among four types of STP of matrices and vectors are studied. To begin with, we focus on STP of matrices and column vectors.

Theorem 3. Given $A \in \mathscr{M}_{m \times n}$ and $x \in \mathscr{V}_{p}$. Suppose $t=[n, p]$ is the least common multiple of $n$ and $p$. Then, the following results hold:

(1) $A \vec{\rtimes}_{1} x=\sum_{i=1}^{t / p} \operatorname{Col}_{i}\left(A \rtimes_{1} x\right)$

(2) $A \vec{\rtimes}_{2} x=(n / t) 1_{t / n} \otimes\left(\sum_{i=1}^{t / n} B l k_{i}\left(A \vec{\rtimes}_{1} x\right)\right)$, where $B l k_{i}$ $\left(A \vec{\rtimes}_{1} x\right)$ is the $i$-th block of $A \vec{\rtimes}_{1} x$ via dividing $A \vec{\rtimes}_{1} x$ into $(t / n)$ equal blocks by rows

(3) $A \rtimes_{2} x=(p / t) 1_{t / p}^{T} \otimes\left(A \vec{\rtimes}_{2} x\right)$
Proof

(1) Since $1_{n}=\sum_{i=1}^{n} \operatorname{Col}_{i}\left(I_{n}\right)$, we have $1_{n} \otimes x=\sum_{i=1}^{n}$ $\operatorname{Col}_{i}\left(I_{n}\right) \otimes x=\sum_{i=1}^{n}\left(\operatorname{Col}_{i}\left(I_{n} \otimes x\right)\right)$ for each $x \in \mathscr{V}_{p}$. According to definitions of MM-1 STP and MV-1 STP, one derives

$$
\begin{aligned}
& A \vec{\rtimes}_{1} x \\
& =\left(I_{t / n} \otimes A\right)\left(1_{t / p} \otimes x\right)=\left(I_{t / n} \otimes A\right)\left(\sum_{i=1}^{t / p} \operatorname{Col}_{i}\left(I_{t / p} \otimes x\right)\right) \\
& =\sum_{i=1}^{t / p} \operatorname{Col}_{i}\left(\left(I_{t / p} \otimes A\right)\left(I_{t / p} \otimes x\right)\right)=\sum_{i=1}^{t / p} \operatorname{Col}_{i}\left(A \rtimes_{1} x\right) .
\end{aligned}
$$

(2) Divide $I_{t / n} \otimes A$ into $t / n$ equal blocks by rows. The $i$-th block of $I_{t / n} \otimes A$ is denoted by $B l k_{i}\left(I_{t / n} \otimes\right.$ $A)=\left(0_{m \times n}, \ldots, A, \ldots, 0_{m \times n}\right)$. It is easy to see that $1_{t / n}^{T} \otimes A=\sum_{i=1}^{t / n} B l k_{i}\left(I_{t / n} \otimes A\right)$ holds. Thus, one gets

$$
\begin{aligned}
& \left(1_{t / n \times t / n} \otimes A\right)\left(1_{t / p} \otimes x\right) \\
& =\left(1_{t / n} \otimes 1_{t / n}^{T} \otimes A\right)\left(1_{t / p} \otimes x\right)=1_{t / n} \otimes\left(\left(1_{t / n}^{T} \otimes A\right)\left(1_{t / p} \otimes x\right)\right)=1_{t / n} \otimes\left(\left(\sum_{i=1}^{t / n} B l k_{i}\left(I_{t / n} \otimes A\right)\right)\left(1_{t / p} \otimes x\right)\right) \\
& =1_{t / n} \otimes \sum_{i=1}^{t / n} B l k_{i}\left(\left(I_{t / n} \otimes A\right)\left(1_{t / p} \otimes x\right)\right)=1_{t / n} \otimes \sum_{i=1}^{t / n} B l k_{i}\left(A \vec{\rtimes}_{1} x\right) .
\end{aligned}
$$

Combined with the definition of MV-2 STP, we can draw the following conclusion:

$$
A \vec{\rtimes}_{2} x=\left(J_{t / n} \otimes A\right)\left(1_{t / p} \otimes x\right)=\frac{n}{t}\left(1_{t / n \times t / n} \otimes A\right)\left(1_{t / p} \otimes x\right)=\frac{n}{t} 1_{t / n} \otimes \sum_{i=1}^{t / n} B l k_{i}\left(A \vec{\rtimes}_{1} x\right) .
$$

(3) From the definition of MM-2 STP, it is not hard to compute 


$$
\begin{aligned}
& A \rtimes_{2} x \\
& =\left(J_{t / n} \otimes A\right)\left(J_{t / p} \otimes x\right)=\frac{p}{t}\left(J_{t / n} \otimes A\right)\left(1_{t / p}^{T} \otimes 1_{t / p} \otimes x\right) \\
& =\frac{p}{t} 1_{t / p}^{T} \otimes\left(\left(J_{t / n} \otimes A\right)\left(1_{t / p} \otimes x\right)\right)=\frac{p}{t} 1_{t / p}^{T} \otimes\left(A \vec{\rtimes}_{2} x\right) .
\end{aligned}
$$

Based on Theorem 3, another relations among four types of STP of matrices and column vectors can be introduced.

Corollary 1. Given $A \in \mathscr{M}_{m \times n}$ and $x \in \mathscr{V}_{p}$. Suppose $t=[n, p]$ is the least common multiple of $n$ and $p$. Relations among all types of STP of $A$ and $x$ are shown as follows:

(1) $A \vec{\rtimes}_{2} x=(n / t) 1_{t / n} \otimes\left(\sum_{i=1}^{t / n} \sum_{j=1}^{t / p} B l k_{i}\left(\operatorname{Col}_{j}\left(A \rtimes_{1} x\right)\right)\right)$, where $B_{1}(M)$ is the $i$-th block of $M$ via dividing $M$ into $t / n$ equal blocks by rows, $M=\operatorname{Col}_{j}\left(A \rtimes_{1} x\right)$.

(2) $A \rtimes_{2} x=\left(n p / t^{2}\right) 1_{t / n \times t / p} \otimes\left(\sum_{i=1}^{t / n} B l k_{i}\left(A \vec{\rtimes}_{1} x\right)\right)$, where $B l k_{i}\left(A \vec{\rtimes}_{1} x\right)$ is the $i$-th block of $A \vec{\rtimes}_{1} x$ via dividing $A \vec{\rtimes}_{1} x$ into $t / n$ equal blocks by rows.

(3) $A \rtimes_{2} x=\left(n p / t^{2}\right) 1_{t / n \times t / p} \otimes\left(\sum_{i=1}^{t / n} \sum_{j=1}^{t / p} B l k_{i}\left(\operatorname{Col}_{j}\left(A \rtimes_{1}\right.\right.\right.$ $x))$ ), where $B l k_{i}(M)$ is the $i$-th block of $M$ via dividing $M$ into $t / n$ equal blocks by rows, $M=\operatorname{Col}_{j}\left(A \rtimes_{1} x\right)$.
The following theorem shows different results between STP of column vectors and matrices and STP of matrices and column vectors.

Theorem 4. Given $A \in \mathscr{M}_{m \times n}$. Let $x \in \mathscr{V}_{p}$ be a column vector. Then, the following results hold:

$$
\begin{aligned}
& \text { (1) } x \vec{\rtimes}_{1} A=x \rtimes_{1} A=A \otimes x \\
& \text { (2) } x \vec{\rtimes}_{2} A=x \rtimes_{2} A=(1 / m) 1_{m} \otimes\left(\sum_{i=1}^{m} \operatorname{Row}_{i}(A) \otimes x\right)
\end{aligned}
$$

Proof

(1) Since $\quad x \vec{\rtimes}_{1} A=\left(I_{m} \otimes x\right) A=\left(\left(I_{m} \otimes x\right) \quad \operatorname{Col}_{1}(A)\right.$ $\left.\cdots\left(I_{m} \otimes x\right) \operatorname{Col}_{n}(A)\right)$, one can see easily that $x \vec{\rtimes}_{1} A=$ $A \otimes x$ holds according to the first item of Theorem 1 . Moreover, we can conclude that $x \rtimes_{1} A=A \otimes x$ is true.

(2) Similarly, $\quad x \rtimes_{2} A=\left(J_{m} \otimes \quad x\right) A=\left(\left(J_{m} \otimes x\right)\right.$ $\left.\mathrm{Col}_{1}(A) \cdots\left(J_{m} \otimes x\right) \operatorname{Col}_{n}(A)\right)$ holds. From the second item of Theorem 1, we derive $\left(J_{m} \otimes x\right) \operatorname{Col}_{i}(A)=(1 / m) \sum_{j=1}^{m} A_{j i}\left(1_{m} \otimes x\right) . \quad$ Thus, one computes

$$
\begin{aligned}
& x \rtimes_{2} A \\
& =\left(\left(J_{m} \otimes x\right) \operatorname{Col}_{1}(A) \cdots\left(J_{m} \otimes x\right) \operatorname{Col}_{n}(A)\right) \\
& =\frac{1}{m}\left(\sum_{j=1}^{m} A_{1 i}\left(1_{m} \otimes x\right) \cdots \sum_{j=1}^{m} A_{n i}\left(1_{m} \otimes x\right)\right)=\frac{1}{m} 1_{m} \otimes\left(\sum_{i=1}^{m} \operatorname{Row}_{i}(A) \otimes x\right) .
\end{aligned}
$$

Based on the definition of MV-2 STP, we also get

$$
x \vec{\rtimes}_{2} A=\frac{1}{m} 1_{m} \otimes\left(\sum_{i=1}^{m} \operatorname{Row}_{i}(A) \otimes x\right) .
$$

Next, we investigate algebraic relations among four types of STP of matrices and row vectors.

Theorem 5. Given $A \in \mathscr{M}_{m \times n}$. Let $x^{T} \in \mathscr{V}_{p}$ be a column vector. Then, the following results hold:

(1) $A \rtimes_{1} x=A \otimes x$

(2) $A \vec{\rtimes}_{2} x=A \vec{\rtimes}_{1} x=x \rtimes_{1}\left(\sum_{i=1}^{n} \operatorname{Col}_{i}(A) \otimes I_{p}\right)$

(3) $A \rtimes_{2} x=(1 / n) 1_{n}^{T} \otimes\left(A \vec{\rtimes}_{2} x\right)=$

$(1 / n) 1_{n}^{T} \otimes\left(x \rtimes_{1}\left(\sum_{i=1}^{n} \operatorname{Col}_{i}(A) \otimes I_{p}\right)\right)$

Proof. (1) It is not hard to find $A \rtimes_{1} x=A$ $\left(I_{n} \otimes x\right)=\left(\left(\operatorname{Row}_{1}(A) I_{n} \otimes x\right)^{T} \cdots\left(\operatorname{Row}_{m}(A)\right.\right.$
$\left.\left.\left(I_{n} \otimes x\right)\right)^{T}\right)^{T}$. The first item of Theorem 2 shows that $\operatorname{Row}_{i}(A)\left(I_{n} \otimes x\right)=\operatorname{Row}_{i}(A) \rtimes_{1}$ $x=\operatorname{Row}_{i}(A) \otimes x$ is true. Hence, $A \rtimes_{1}$ $x=\left(\left(\operatorname{Row}_{1}(A) \otimes x\right)^{T} \cdots\left(\operatorname{Row}_{m}(A) \otimes x\right)^{T}\right)^{T}$ $=A \otimes x$ holds.

(2) By computation direct, we draw the following conclusion:

$$
\begin{aligned}
& A \vec{\rtimes}_{1} x \\
& =A \vec{\rtimes}_{2} x=A\left(1_{n} \otimes x\right)=\sum_{i=1}^{n} \operatorname{Col}_{i}(A) \otimes x \\
& =\left(I_{m} \otimes x\right)\left(\sum_{i=1}^{n} \operatorname{Col}_{i}(A) \otimes I_{p}\right)=x \rtimes_{1}\left(\sum_{i=1}^{n} \operatorname{Col}_{i}(A) \otimes I_{p}\right) .
\end{aligned}
$$

(3) Combining (15) with definitions of STP, one derives the following results: 


$$
\begin{aligned}
& A \rtimes_{2} x \\
& =A\left(J_{n} \otimes x\right)=\frac{1}{n} A\left(1_{n \times n} \otimes x\right)=\frac{1}{n} A\left(1_{n}^{T} \otimes 1_{n} \otimes x\right)=\frac{1}{n} 1_{n}^{T} \otimes\left(A\left(1_{n} \otimes x\right)\right) \\
& =\frac{1}{n} 1_{n}^{T} \otimes\left(A \vec{\rtimes}_{2} x\right)=\frac{1}{n} 1_{n}^{T} \otimes\left(A \vec{\rtimes}_{1} x\right) \\
& =\frac{1}{n} 1_{n}^{T} \otimes\left(x \rtimes_{1}\left(\sum_{i=1}^{n} \operatorname{Col}_{i}(A) \otimes I_{p}\right)\right) .
\end{aligned}
$$

3.3. STP of Two Matrices. Combined with the analysis above, algebraic relations among four types of STP of two matrices are derived easily.

Theorem 6. Given $A \in \mathscr{M}_{m \times n}$ and $B \in \mathscr{M}_{p \times q}$. Suppose $t=$ $[n, p]$ is the least common multiple of $n$ and $p$. Then, the following results hold:

(1) $A \vec{\rtimes}_{1} B=\left(\sum_{i=1}^{t / p} \operatorname{Col}_{i}\left(A \rtimes_{1} B\right) \cdots \sum_{i=(q-1) t / p+1}^{t q / p} \operatorname{Col}_{i}\left(A \rtimes_{1}\right.\right.$ B))

(2) $A \vec{\rtimes}_{2} B=(n / t) 1_{t / n} \otimes\left(\sum_{i=1}^{t / n} B l k_{i}\left(A \vec{\rtimes}_{1} B\right)\right)$, where $B l k_{i}$ $\left(A \vec{\rtimes}_{1} B\right)$ is the $i$-th block of $A \vec{\rtimes}_{1} B$ via dividing $A \vec{\rtimes}_{1} x$ into $t / n$ equal blocks by rows

(3) $A \rtimes_{2} B=(p / t) 1_{t / p}^{T} \otimes\left(A \vec{\rtimes}_{2} B\right)$

Proof. Since proofs of these tree items are similar, we only prove (1).

(1) From equation (5), one finds $A \vec{x}_{1} B=$ $\left(A \vec{\rtimes}_{1} \operatorname{Col}_{1}(B) \cdots A \vec{\rtimes}_{1} \operatorname{Col}_{q}(B)\right)$. Because $A \vec{\rtimes}_{1} \operatorname{Col}_{i}$ $(B)=\sum_{j=1}^{t / p} \operatorname{Col}_{j}\left(A \rtimes_{1} \operatorname{Col}_{i}(B)\right)$ holds according to Theorem 3, it is not difficult to see $A \vec{\rtimes}_{1} B=\left(\sum_{j=1}^{t / p}\right.$ $\left.\mathrm{Col}_{j}\left(A \rtimes_{1} \operatorname{Col}_{1}(B)\right) \cdots \sum_{j=1}^{t / p} \operatorname{Col}_{j}\left(A \rtimes_{1} \operatorname{Col}_{q}(B)\right)\right)$. By calculation, we conclude that

$$
A \vec{\rtimes}_{1} B=\left(\sum_{i=1}^{t / p} \operatorname{Col}_{i}\left(A \rtimes_{1} B\right) \cdots \sum_{i=(q-1) t / p+1}^{t q / p} \operatorname{Col}_{i}\left(A \rtimes_{1} B\right)\right),
$$

which is true.

Similarly, another relation among of four types of STP of two matrices is presented.

Corollary 2. Given $A \in \mathscr{M}_{m \times n}$ and $B \in \mathscr{M}_{p \times q}$. Suppose $t=$ $[n, p]$ is the least common multiple of $n$ and $p$. Relations among all types of STP of $A$ and $B$ are shown as follows:

(1) $A \vec{\rtimes}_{2} B=(n / t) 1_{t / n} \otimes \sum_{i=1}^{t / n} B l k_{i}\left(\sum_{j=1}^{t /} \quad p \operatorname{Col}_{j}\left(A \rtimes_{1} B\right)\right.$ $\left.\cdots \sum_{j=(q-1) t / p+1}^{t q} \mathrm{Col}_{j}\left(A \rtimes_{1} B\right)\right)$, where Blk $k_{i}(M)$ is the $i$-th block of $M$ via dividing $M$ into $t / n$ equal blocks by rows, $\quad M=\left(\sum_{j=1}^{t / p} \operatorname{Col}_{j}\left(A \rtimes_{1} B\right) \cdots \sum_{j=(q-1) t / p+1}^{t q t p} C o l_{j}\right.$ $\left.\left(A \rtimes_{1} B\right)\right)$
(2) $A \rtimes_{2} B=\left(n p / t^{2}\right) 1_{t / n \times t / p} \otimes\left(\sum_{i=1}^{t / n} B l k_{i}\left(A \vec{\rtimes}_{1} B\right)\right)$, where $B k_{i}\left(A \vec{\rtimes}_{1} B\right)$ is the $i$-th block of $A \vec{\rtimes}_{1} B$ via dividing $A \vec{x}_{1} x$ into $t / n$ equal blocks by rows

(3) $A \rtimes_{2} B=\left(n p / t^{2}\right) 1_{t / n \times} t / p \otimes \sum_{i=1}^{t / n} B l k_{i}\left(\sum_{j=1}^{t /} p \operatorname{Col}_{j}(A\right.$ $\left.\left.\rtimes_{1} B\right) \cdots \sum_{j=(q-1) t / p+1}^{t q / p} \operatorname{Col}_{j}\left(A \rtimes_{1} B\right)\right)$, where $B_{l}(M)$ is the $i$-th block of $M$ via dividing $M$ into $t / n$ equal blocks by rows, $M=\left(\sum_{j=1}^{t / p} \operatorname{Col}_{j}\left(A \rtimes_{1} B\right) \ldots\right.$ $\left.\sum_{j=(q-1) t / p+1}^{t q / p} \operatorname{Col}_{j}\left(A \rtimes_{1} B\right)\right)$

\section{Conclusion}

This paper has studied relations among four kinds of right STPs under three cases, containing STP of two vectors, STP of matrices and vectors, and STP of two matrices. The results obtained in this paper have shown the convertibility of these right STPs.

\section{Data Availability}

No data were used to support this study.

\section{Conflicts of Interest}

The authors declare that they have no conflicts of interest.

\section{Acknowledgments}

This work was supported by the grants from the Natural Science Foundation Project of Fujian, China (no. 2020J01795).

\section{References}

[1] D. Cheng, "Semi-tensor product of matrices and its application to morgen's problem," Science China Information Sciences, vol. 44, no. 3, pp. 195-212, 2001.

[2] Y. Zheng, H. Li, and J.-E. Feng, "State-feedback set stabilization of logical control networks with state-dependent delay," Science China Information Sciences, vol. 64, no. 6, p. 169203, 2021 1-169203:3.

[3] Y. Li, J. Li, and J.-e. Feng, "Set controllability of Boolean control networks with impulsive effects," Neurocomputing, vol. 418, pp. 263-269, 2020.

[4] Y. Li, J. Li, and J.-E. Feng, "Output tracking of Boolean control networks with impulsive effects," IEEE Access, vol. 8, pp. 157793-157799, 2020. 
[5] D. Cheng, "On finite potential games," Automatica, vol. 50, no. 7, pp. 1793-1801, 2014.

[6] D. Cheng and T. Liu, "From Boolean game to potential game," Automatica, vol. 96, pp. 51-60, 2018.

[7] C. Li, Y. Xing, F. He, and D. Cheng, "A strategic learning algorithm for state-based games," Automatica, vol. 113, pp. 1-9, 2020.

[8] D. Cheng, J.-e. Feng, and H. Lv, "Solving fuzzy relational equations via semitensor product," IEEE Transactions on Fuzzy Systems, vol. 20, no. 2, pp. 390-396, 2012.

[9] J. Feng, H. Lv, and D. Cheng, "Multiple fuzzy relation and its application to coupled fuzzy control," Asian Journal of Control, vol. 15, no. 5, pp. 1313-1324, 2013.

[10] Y. Wang, C. Zhang, and Z. Liu, "A matrix approach to graph maximum stable set and coloring problems with application to multi-agent systems," Automatica, vol. 48, no. 7 , pp. 1227-1236, 2012.

[11] M. Meng and J. Feng, "A matrix approach to hypergraph stable set and coloring problems with its application to storing problem," Journal of Applied Mathematics, pp. 1-10, 2014.

[12] D. Cheng, "On equivalence of matrices," Asian Journal of Mathematics, vol. 23, no. 2, pp. 257-348, 2019.

[13] D. Cheng, Z. Liu, and H. Qi, "Cross-dimensional linear systems,” 2017, https://arxiv.org/abs/1710.03530.

[14] Q.-1. Zhang, B. Wang, and J.-e. Feng, "Solution and stability of continuous-time cross-dimensional linear systems," Frontiers of Information Technology and Electronic Engineering, vol. 22, no. 2, pp. 210-221, 2021.

[15] J.-e. Feng, B. Wang, and Y. Yu, "On dimensions of linear discrete dimension-unbounded systems," International Journal of Control, Automation and Systems, vol. 19, no. 1, pp. 471-477, 2021.

[16] P. Zhao, H. Guo, Y. Yu, and J. Feng, "On dimensions of dimension-bounded linear systems," Science China Information Sciences, vol. 64, no. 5, pp. 1-3, 2021.

[17] D. Cheng, Z. Liu, Z. Xu, and T. Shen, "Generalised semitensor product of matrices," IET Control Theory and Applications, vol. 14, no. 1, pp. 85-95, 2020.

[18] D. Cheng, Z. Xu, and T. Shen, "Equivalence-based model of dimension-varying linear systems," IEEE Transactions on Automatic Control, vol. 65, no. 12, pp. 5444-5449, 2020.

[19] J. Feng, Y. Li, and J. Zhao, "Four kinds of semi-tensor products and their relationships," Journal of Liaocheng University (Nat. Sci.), vol. 33, no. 4, pp. 1-7, 2020, in Chinese.

[20] D. Cheng, H. Qi, and Z. Li, Analysis and Control of Boolean Networks: A Semi-tensor Product Approach, Springer, London, UK, 2011.

[21] D. Cheng, H. Qi, and Y. Zhao, An Introduction to Semi-tensor Product of Matrices and its Application, World Scientific, Singapore, 2011. 